\title{
No role for neutrons, muons and solar neutrinos in the DAMA annual modulation results
}

\author{
R. Bernabei ${ }^{1,2, a}$, P. Belli ${ }^{2}$, F. Cappella ${ }^{3}$, V. Caracciolo ${ }^{3}$, R. Cerulli ${ }^{3}$, C. J. Dai ${ }^{4}$, A. d'Angelo ${ }^{5,6}$, S. d'Angelo ${ }^{1,2}$, \\ A. Di Marco ${ }^{1,2}$, H. L. He ${ }^{4}$, A. Incicchitti ${ }^{5,6}$, H. H. Kuang ${ }^{4}$, X. H. Ma ${ }^{4}$, F. Montecchia ${ }^{2,7}$, X. D. Sheng ${ }^{4}$, R. G. Wang ${ }^{4}$, \\ Z. P. $\mathbf{Y e}^{4,8}$ \\ ${ }^{1}$ Dip. di Fisica, Università di Roma "Tor Vergata”, 00133 Rome, Italy \\ 2 INFN, sez. Roma "Tor Vergata", 00133 Rome, Italy \\ ${ }^{3}$ Laboratori Nazionali del Gran Sasso, INFN, Assergi, Italy \\ ${ }^{4}$ Key Laboratory of Particle Astrophysics, Institute of High Energy Physics, Chinese Academy of Sciences, \\ P.O. Box 918/3, Beijing 100049, China \\ 5 Dip. di Fisica, Università di Roma "La Sapienza", 00185 Rome, Italy \\ ${ }^{6}$ INFN, sez. Roma, 00185 Rome, Italy \\ ${ }^{7}$ Dip. di Ingegneria Civile e Ingegneria Informatica, Università di Roma "Tor Vergata", 00133 Rome, Italy \\ ${ }^{8}$ University of Jing Gangshan, Ji' an, Jiangxi, China
}

Received: 15 September 2014 / Accepted: 24 November 2014 / Published online: 9 December 2014

(C) The Author(s) 2014. This article is published with open access at Springerlink.com

\begin{abstract}
This paper summarizes in a simple and intuitive way why the neutrons, the muons and the solar neutrinos cannot give any significant contribution to the DAMA annual modulation results. A number of these elements have already been presented in individual papers; they are recalled here together with few simple considerations which demonstrate the incorrectness of the claim reported in Davis (PRL 113:081302, 2014).
\end{abstract}

\section{Introduction}

The DAMA/LIBRA experiment - as the former DAMA/NaI - at the Gran Sasso underground laboratory (LNGS) of the INFN is investigating the presence of the dark matter (DM) particles in the galactic halo by exploiting the model independent DM annual modulation signature. This DM signature is very distinctive since the effect induced by DM particles must simultaneously satisfy all the following requirements: (1) the event rate must contain a component modulated according to a cosine function; (2) with period equal to 1 year; (3) with a phase roughly around June 2nd in case of usually adopted halo models (slight variations may occur in case of presence of non thermalized DM components in the halo); (4) this modulation must be present only at low energy, where DM particles can induce signals; (5) it must be present only for single-hit events (where just a single detector, in a multidetector set-up, actually "fires"), since the probability that

\footnotetext{
a e-mail: rita.bernabei@roma2.infn.it
}

DM particles experience multiple interactions is negligible; (6) the modulation amplitude in the region of maximal sensitivity has to be $\lesssim 7 \%$ (it may also be significantly larger in some particular scenarios).

To mimic such a signature spurious effects or side reactions should be able not only to account for the observed modulation amplitude but also to simultaneously satisfy all the requirements of the signature; thus, no other effect investigated so far in the field of rare processes offers a so stringent and unambiguous signature.

In addition, let us note that neutrons, muons and solar neutrinos are not a competing background when the DM annual modulation signature is investigated, since in no case they can mimic this signature. Moreover, the sensitivity of the DAMA experiments - in contrast to others - is not restricted only to DM candidates giving rise just to nuclear recoils through elastic scatterings on target nuclei.

Let us now briefly describe the DAMA/LIBRA experiment [1], recalling its model independent annual modulation results [2-4]. DAMA/LIBRA is made of 25 highly radiopure $\mathrm{NaI}(\mathrm{Tl})$ crystal scintillators, each one of $9.70 \mathrm{~kg}$ mass and size of $(10.2 \times 10.2 \times 25.4) \mathrm{cm}^{3}$, in a 5-rows 5-columns matrix. The detectors are housed in a low-radioactivity sealed copper box installed in the center of a passive shield made by $10 \mathrm{~cm}$ of OFHC low radioactive copper, $15 \mathrm{~cm}$ of low radioactive lead, $1.5 \mathrm{~mm}$ of cadmium and about $10-40 \mathrm{~cm}$ of polyethylene/paraffin (thickness fixed by the available space); moreover, about $1 \mathrm{~m}$ concrete (made from the Gran Sasso rock material) almost fully surrounds (mostly outside the barrack) 
this passive shield, acting as a further neutron moderator. In particular, the neutron shield reduces by a factor larger than one order of magnitude the environmental thermal neutrons flux [2]. The copper box is continuously maintained in HP Nitrogen atmosphere in slight overpressure with respect to the external environment; it is part of the 3-levels sealing system which prevents environmental air reaching the detectors. The DAMA/LIBRA-phase1 exposure (1.04 ton $\times$ year) has been collected during seven annual cycles [2-4]. Considering also the former DAMA/NaI $[5,6]$, the total exposure collected over 14 annual cycles is 1.33 ton $\times$ year.

A clear modulation is present in the (2-6) $\mathrm{keV}$ single-hit events and fulfills all the requirements of the DM annual modulation signature; in particular, no modulation is observed either above $6 \mathrm{keV}$ or in the (2-6) $\mathrm{keV}$ multiple-hits events [2-4].

The data provide a model independent evidence of the presence of DM particles in the galactic halo at 9.3 $\sigma$ C.L. on the basis of the investigated DM signature. In particular, with the cumulative exposure the modulation amplitude of the single-hit events in the (2-6) keV energy interval, measured in $\mathrm{NaI}(\mathrm{Tl})$ target, is $(0.0112 \pm 0.0012) \mathrm{cpd} / \mathrm{kg} / \mathrm{keV}$; the measured phase is (144 \pm 7 ) days (corresponding to May 24 \pm 7 days) and the measured period is $(0.998 \pm 0.002)$ year, values well in agreement with those expected for the DM particles.

Careful investigations on absence of any significant systematics or side reaction able to account for the measured modulation amplitude and to simultaneously satisfy all the requirements of the signature have been quantitatively carried out (see e.g. [1-8], and references therein); none has been found or suggested by anyone over more than a decade. In particular, the cases of the neutrons of whatever origin, and of the muons have been deeply investigated.

This paper summarizes in a simple and intuitive way why the neutrons, the muons and the solar neutrinos cannot significantly contribute to the DAMA observed annual modulation signal; some of the already-published arguments [1-8] are also recalled here. Afterwards, we demonstrate through few simple considerations the incorrectness of the claim reported in [9].

\section{Neutrons, muons and solar neutrinos at LNGS}

In the following we discuss quantitatively the background induced by neutrons, muons and solar neutrinos in DAMA/LIBRA.

\subsection{The neutron flux at LNGS}

The total flux of neutrons is given by the sum of the thermal, the epithermal, and the fast components; the latter can be written as:
$\Phi_{\text {fast }}^{(n)}=\Phi_{\text {fiss, } \alpha \rightarrow n}^{(n)}+\Phi_{\mu \rightarrow n}^{(n)}+\Phi_{\nu \rightarrow n}^{(n)}$

where: (1) $\Phi_{\text {fiss, } \alpha \rightarrow n}^{(n)}$, the dominant component, is due to neutrons from fissioning elements and from $(\alpha, n)$ reactions (neutron energy roughly from 1 to $10 \mathrm{MeV}$ ); (2) $\Phi_{\mu \rightarrow n}^{(n)}$ is due to neutrons generated by $\mu$ interactions (neutron energy distribution with a long tail up to GeV's [10-12]); (3) $\Phi_{\nu \rightarrow n}^{(n)}$ is due to neutrons generated by solar neutrinos interactions (neutron energy roughly few $\mathrm{MeV}$ ). The possible yearly variation, if any, of each component $k$ can be pointed out by simply considering a cosine-like first term approximation:

$\Phi_{k}^{(n)}=\Phi_{0, k}^{(n)}\left(1+\eta_{k} \cos \omega\left(t-t_{k}\right)\right)$,

neglecting higher order harmonics; here $\omega=2 \pi /(1$ year), $\eta_{k}$ is the relative modulation amplitude and $t_{k}$ the phase. The neutrons in the Gran Sasso caverns were measured many times by several authors [13-18]. The measurements are largely compatible among them; in particular, the flux of thermal neutron is $\simeq 1.08 \times 10^{-6} \mathrm{n} \mathrm{cm}^{-2} \mathrm{~s}^{-1}$ [15], the flux of epithermal neutron is $\simeq 2 \times 10^{-6} \mathrm{n} \mathrm{cm}^{-2} \mathrm{~s}^{-1}$ [15], and the flux of fast neutron is $\simeq 0.9 \times 10^{-7} \mathrm{n} \mathrm{cm}^{-2} \mathrm{~s}^{-1}$ [17]. In addition, the measured flux of neutrons with energy above 10 $\mathrm{MeV}$, where the muon-induced neutrons mostly contribute, is $\simeq 0.5 \times 10^{-9} \mathrm{n} \mathrm{cm}^{-2} \mathrm{~s}^{-1}[15,18]$. It is worth noting that, at present, no compelling evidence of any time variation of the thermal, epithermal and fast neutrons is available (for details see $[2,7,8])$. In the following calculations we conservatively adopt $\eta_{k}=0.1$ as done in $[2,7,8]$; thus, since $\eta_{k} \ll 0.1$ the derived values are conservative upper limits.

We stress that these measurements regard all neutrons of whatever origin and, therefore, even those induced by muons and by solar neutrinos. In particular, $\Phi_{0, \mu \rightarrow n}^{(n)}$ and $\Phi_{0, v \rightarrow n}^{(n)}$ are less (or more correctly, much less) than the total flux of neutrons; to estimate them, we adopt here the values reported in [9] for the induced neutron rate production at $\mathrm{LNGS}^{1}: r_{\mu} \approx$ $0.86 \mathrm{n} / \mathrm{day} / \mathrm{m}^{3}$ from muons and $r_{v} \approx 0.09 \mathrm{n} / \mathrm{day} / \mathrm{m}^{3}$ from solar neutrinos. The $r_{\mu}$ value is within the range obtained in [7] for neutrons produced by muons interactions in the surrounding rocks, considering: (1) the muon flux, $\Phi_{0}^{(\mu)}$ (see later); (2) the neutron yield in the rock $Y_{\text {rock }} \simeq(1-7) \times 10^{-4}$ neutrons per muons per $\mathrm{g} / \mathrm{cm}^{2}$ [12]; (3) the density of the rock at LNGS, $\rho_{\text {rock }} \simeq 2.7 \mathrm{~g} / \mathrm{cm}^{3}$ (see e.g. [19]).

Besides the rock, a contribution can also arise from high $-Z$ materials around the $\mathrm{NaI}(\mathrm{Tl})$ detectors, in particular the lead shield; we will discuss this item in the following.

\footnotetext{
${ }^{1}$ From [9]: (1) the muon-induced neutron rate is $R_{\mu} \simeq 10^{-34} n V$ neutrons/s; (2) the neutrino-induced neutron rate is $R_{v} \simeq 10^{-35} n V$ neutrons/s; where: $n$ is the number density of the target $\simeq 10^{29} \mathrm{~m}^{-3}$ and $V$ is the target volume. Therefore, the induced neutron rate production per target volume unit is: $r_{\mu}=R_{\mu} / V \approx 0.86 \mathrm{n} / \mathrm{day} / \mathrm{m}^{3}$ for muon-induced interactions, and $r_{v}=R_{v} / V \approx 0.09 \mathrm{n} / \mathrm{day} / \mathrm{m}^{3}$ for solar neutrino-induced interactions.
} 
A right calculation of the effective volume, $V_{\text {eff }}$, over which the neutrons are produced and reach the detectors needs a simulation. For the accuracy needed here, it is sufficient to consider a simple model taking into account only the geometry of the set-up and the mean free path of the produced neutrons. In particular, the DAMA/LIBRA setup (about $51 \times 51 \times 25.4 \mathrm{~cm}^{3}$, that is its volume is $\left.V_{L I B R A}=0.066 \mathrm{~m}^{3}\right)$ is considered at the center of a sphere of material where the neutrons are evenly and isotropically generated.

For a mean free path of the neutrons conservatively assumed about $2.6 \mathrm{~m}$ (as in [9]), and considering a sphere with $10 \mathrm{~m}$ radius (about 4 times the neutrons mean free path), that is a volume $V=4200 \mathrm{~m}^{3}$, the mean probability that the induced neutrons reach the DAMA/LIBRA setup can be calculated by the simulation to be: $P=0.016 \%$; that is the effective volume is: $V_{\text {eff }}=V \times P=0.7 \mathrm{~m}^{3}$.

The simulation also provides $\Phi_{0, \mu \rightarrow n}^{(n)} \simeq 3 \times 10^{-9} \mathrm{n} \mathrm{cm}^{-2}$ $\mathrm{s}^{-1}$, and $\Phi_{0, v \rightarrow n}^{(n)} \simeq 3 \times 10^{-10} \mathrm{n} \mathrm{cm}^{-2} \mathrm{~s}^{-1}$, two orders of magnitude lower than the measured total one (given above); $\Phi_{0, \mu \rightarrow n}^{(n)}$ is compatible with the above-mentioned measurements at LNGS for neutron energy above $10 \mathrm{MeV}[15,18]$, and with the expectations of [12], where a total muon-induced neutron flux: $2.7 \times 10^{-9} \mathrm{n} \mathrm{cm}^{-2} \mathrm{~s}^{-1}$, has been calculated for LNGS.

A simple analytical calculation leads to the same conclusions; taking into account that every point around the DAMA/LIBRA setup can be a source of neutrons induced by muons (neutrinos) with a rate $r_{\mu(v)}$ and conservatively assuming a mean free path $\lambda_{0}=2.6 \mathrm{~m}$, one can write:

$$
\begin{aligned}
\Phi_{0, \mu(\nu) \rightarrow n}^{(n)} & =\int_{V} \frac{d \Phi(\mathbf{r})}{d V} d^{3} r=\int_{V} \frac{r_{\mu(\nu)}}{4 \pi r^{2}} e^{-r / \lambda_{0}} d^{3} r \\
& =r_{\mu(v)} \int_{0}^{\infty} e^{-r / \lambda_{0}} d r=r_{\mu(\nu)} \lambda_{0} .
\end{aligned}
$$

So that, $\Phi_{0, \mu \rightarrow n}^{(n)}=r_{\mu} \lambda_{0} \simeq 2.6 \times 10^{-9} \mathrm{n} \mathrm{cm}^{-2} \mathrm{~s}^{-1}$ and $\Phi_{0, v \rightarrow n}^{(n)}=r_{v} \lambda_{0} \simeq 2.6 \times 10^{-10} \mathrm{n} \mathrm{cm}^{-2} \mathrm{~s}^{-1}$, respectively, confirming the results of the simulation.

Let us evaluate also the amount of neutrons from muons interactions in the lead shield. For high $-Z$ materials the neutron yield is larger; in particular, for lead target the neutron yield is [20]: $Y \simeq 1.3 \times 10^{-3}$ neutrons per muons per $\mathrm{g} / \mathrm{cm}^{2}$, and $r_{\mu}$ becomes $\simeq 29 \mathrm{n} / \mathrm{day} / \mathrm{m}^{3}$. Considering that the total volume of the lead shield of DAMA/LIBRA is $V_{P b} \simeq 1 \mathrm{~m}^{3}$, and $P=4.5 \%$ by the simulation, one can obtain an effective volume for the lead shield equal to $V_{P b} \times P=0.045 \mathrm{~m}^{3}$. Thus, the corresponding neutron flux is $\simeq 6 \times 10^{-9} \mathrm{n} \mathrm{cm}^{-2}$ $\mathrm{s}^{-1}$.

All the neutron fluxes are reported in Table 1.

\subsection{The muons at LNGS}

The surviving muon flux, $\Phi^{(\mu)}$, has been measured deeply underground in the Gran Sasso Laboratory at $3600 \mathrm{~m}$ w.e. depth by various experiments with very large exposures [2126]; its mean value is $\Phi_{0}^{(\mu)} \simeq 20$ muons $\mathrm{m}^{-2} \mathrm{~d}^{-1}$ [21], that is about a factor $10^{6}$ lower than measured at sea level. The measured average single muon energy at LNGS is [270 \pm $3($ stat $) \pm 18($ syst $)] \mathrm{GeV}[12,27] . \mathrm{A} \simeq 2 \%$ yearly variation of the muon flux was firstly measured years ago by MACRO; an extensive discussion about the muon flux variation along the year measured by the experiments at LNGS has been reported in [7]. For the purpose of this paper we assume that:

$\Phi^{(\mu)}=\Phi_{0}^{(\mu)}\left(1+\eta^{(\mu)} \cos \omega\left(t-t^{(\mu)}\right)\right) ;$

$t^{(\mu)}$ at LNGS location is at end of June (or later depending on each year; see e.g. [7] and references therein), and the relative modulation amplitude $\eta^{(\mu)}$ is $\simeq 0.0129 \pm 0.0007$ [25].

\subsection{Solar neutrinos at LNGS}

The total solar neutrino flux at LNGS is well established [28]. Its time variability, experimentally pointed out - still with modest C.L. - by Super-Kamiokande (fiducial volume of 22.5 kton) [29,30], SNO (1000 tons) [31], and Borexino (fiducial volume 100-150 tons) [32], is due to the different Sun-Earth distance along the year; so the relative modulation amplitude is twice the eccentricity of the Earth orbit and the phase is at the perihelion. Thus, the total neutrino flux (from every source: pp, ${ }^{7} \mathrm{Be},{ }^{8} \mathrm{~B}$, pep, ... ) can be written as:

$\Phi^{(\nu)}=\Phi_{0}^{(v)}\left(1+\eta^{(v)} \cos \omega\left(t-t^{(\nu)}\right)\right)$,

where the phase, $t^{(v)}$, corresponds to Jan. 4 th and the relative modulation amplitude $\eta^{(v)}$ is 0.03342 .

The values of the parameters in Eqs. 2, 3 and 4 are reported in Table 1.

\section{Counting rate in DAMA/LIBRA induced by neutrons, muons and solar neutrinos}

The counting rate of the DAMA/LIBRA detectors for singlehit events, in the (2-6) keV energy region due to neutrons, muons and solar neutrinos can be written as the sum of seven contributions due to:

1. thermal neutrons $\left(R_{\text {thermal } n}\right)$;

2. epithermal neutrons $\left(R_{\text {epith.n }}\right)$;

3. fast neutrons due to fissioning elements and to $(\alpha, n)$ reactions $\left(R_{f i s s, \alpha \rightarrow n}\right)$;

4. fast neutrons generated by $\mu$ interactions $\left(R_{\mu \rightarrow n}\right)$; 


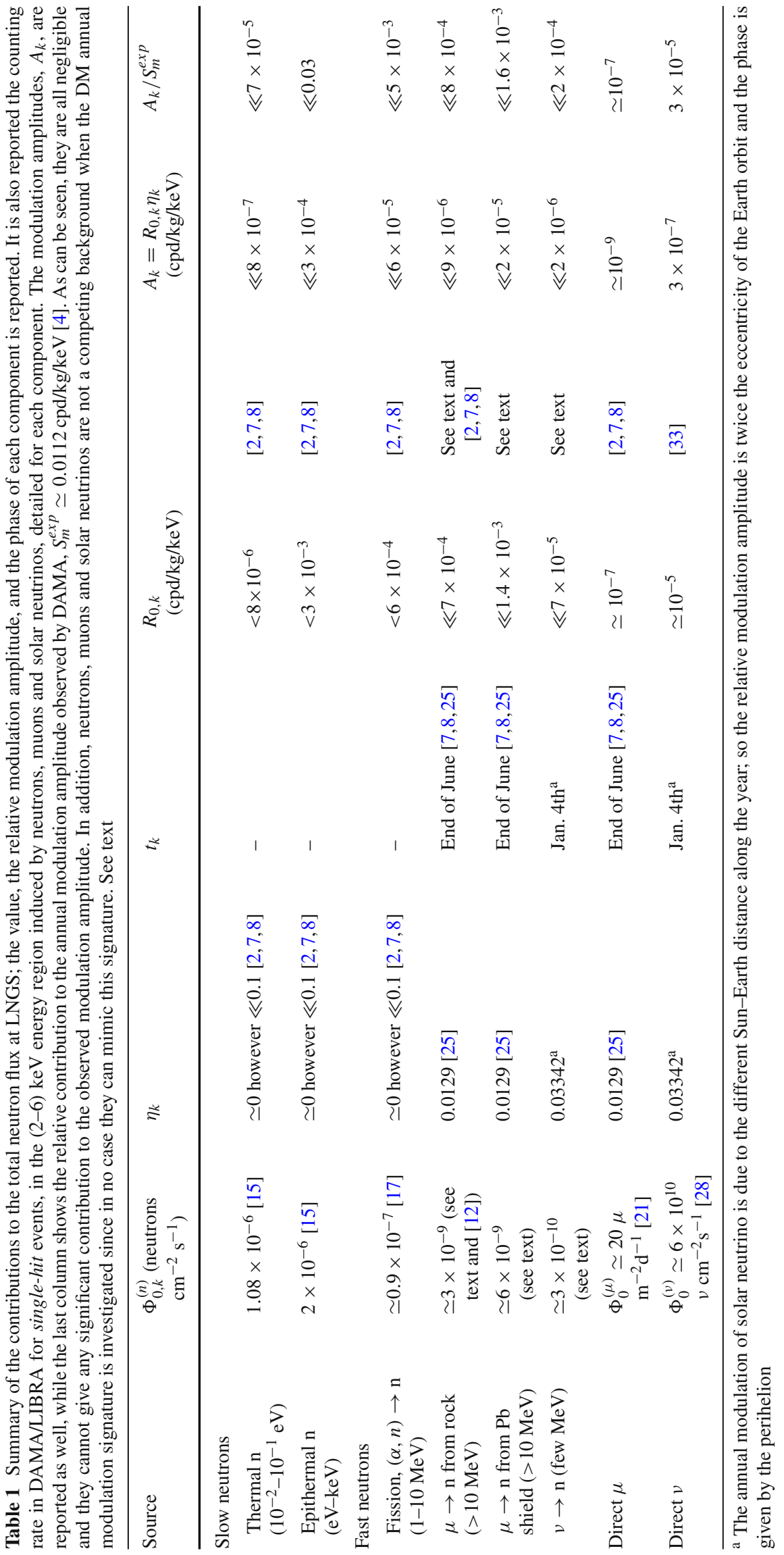


5. fast neutrons generated by solar neutrinos interactions $\left(R_{v \rightarrow n}\right)$;

6. direct interactions of muons $\left(R_{\text {direct } \mu}\right)$;

7. direct interactions of solar neutrinos $\left(R_{\text {direct }} v\right)$.

The possible yearly modulation, if any, of each component $k$ can be described as:

$R_{k}=R_{0, k}\left(1+\eta_{k} \cos \omega\left(t-t_{k}\right)\right)$.

The modulation amplitude of the component $k$ is $A_{k}=$ $R_{0, k} \eta_{k}$. The known parameters are reported in Table 1 .

In particular, the $R_{0, k}$ from neutrons and from muons were calculated in $[2,7,8]$, while the one from "direct" neutrino interactions was calculated in [33]. As concern the $R_{0, k}$ from muon-induced and neutrino-induced neutrons, they can be calculated by the simplified model used above. For simplicity, we do not take into account e.g. the further reducing effect of the neutron shield of DAMA/LIBRA. One has: (1) neutrons directly induced in the DAMA/LIBRA setup $=r_{\mu(v)} V_{L I B R A}=0.06(0.006)$ neutrons/day for muon(neutrino-) induced events; (2) neutrons induced in the surrounding sphere $=r_{\mu(v)} V_{e f f}=0.58(0.06)$ neutrons/day for muon- (neutrino-) induced events.

Therefore, even for detection efficiency equal to 1 , the rate from muon-induced neutrons in DAMA/LIBRA cannot exceed $0.64 \mathrm{cpd}$; more likely the rate is $\ll 0.64 \mathrm{cpd}$. Maximizing the effect, and even assuming that such counts are only single-hit and in the $2-6 \mathrm{keV}$ energy region, the counting rate $\ll \frac{0.64 \mathrm{cpd}}{4 \mathrm{keV} 242.5 \mathrm{~kg}} \simeq 7 \times 10^{-4} \mathrm{cpd} / \mathrm{kg} / \mathrm{keV}$ can be obtained (to be compared with the single-hit total rate of the DAMA detectors that is around $1 \mathrm{cpd} / \mathrm{kg} / \mathrm{keV}$ [1]). One order of magnitude less is from neutrino-induced neutrons.

We note that the modulation amplitude for the case of neutrons induced by muons (see Table 1) is well compatible with the value estimated using different approach $[2,7,8]$ : $\ll(3-24) \times 10^{-6} \mathrm{cpd} / \mathrm{kg} / \mathrm{keV}$.

All these values are reported in Table 1. The last column shows for each component the relative contribution to the annual modulation amplitude observed by DAMA/LIBRA. As can be seen, they are all negligible and they cannot give any significant contribution to the observed modulation amplitude.

In addition, it is worth noting that neutrons, muons and solar neutrinos cannot mimic the annual modulation signature, since they would induce e.g. variations in all the energy spectrum, variation in the multiple-hit events,...consistently not observed in DAMA data $[2-4,7,8]$.

\section{Four simple arguments against the claim of [9]}

The arguments summarized above clearly demonstrate that the claim in [9] is incorrect. In this Section we report in addi- tion just few simple underlying considerations that would already have been enough to clearly demonstrate the incorrectness of the claim in [9].

The paper of [9] reports about a fit on the residuals of DAMA/LIBRA annual modulation result $[1-4,7,8]$, using the following function:

$A_{\mu+\nu}=A_{\nu} \cos \left(\omega\left(t-\phi_{\nu}\right)\right)+A_{\mu} \cos \left(\omega\left(t-\phi_{\mu}\right)\right) ;$

where the two contributions are claimed due to neutrons produced by solar neutrino and muon interactions, respectively. The frequency and phases are constrained to: $\omega=2 \pi /(1$ year), $\phi_{\nu} \simeq$ January 4 th, and $\phi_{\mu} \simeq$ end of June (see also $[7,8]$ ), while the two modulation amplitudes, $A_{\nu}$ and $A_{\mu}$, are considered as free parameters of the fit. This "mathematical" exercise produces two "big" modulation amplitudes since a sort of cancellation occurs between the two effects, having quasi-opposite phases.

But this "mathematical" exercise does not represent a physical possibility for many reasons, as shown in the following.

4.1 Neutrons from whatever origin (even from muons and from solar neutrinos) cannot give any relevant contribution to the DAMA modulation effect

It was already demonstrated (see e.g. $[2,7,8]$ ) that the neutrons - independently of their origin - cannot give any relevant contribution to the DAMA modulation effect. Therefore, any hypothetical contribution of neutrons - and in particular those from muons and solar neutrinos - to the DAMA annual modulation effect is absolutely negligible, if any. This has been summarized above and shown in Table 1.

In addition, as mentioned above, it is worth noting that neutrons of whatever origin cannot mimic the DM annual modulation signature.

\subsection{Results of the fit reported in [9] lead to erroneous claims}

The fitting procedure, reported in [9], on the single-hit experimental residuals measured by DAMA leads to the absurd conclusion that the fitted modulation amplitude $A_{\mu}$ is of the same amount as $A_{\nu}$.

In particular, the fitting procedure in [9] yields to $A_{v} \simeq$ $0.039 \mathrm{cpd} / \mathrm{kg} / \mathrm{keV}$ and $A_{\mu} \simeq 0.047 \mathrm{cpd} / \mathrm{kg} / \mathrm{keV}$; using the relative modulation amplitudes of solar neutrinos and muons given above, one can easily determine in such hypothesis the respective contributions to the single-hit total rate of the DAMA/LIBRA detectors. They would be $0.039 / 0.03342=$ $1.17 \mathrm{cpd} / \mathrm{kg} / \mathrm{keV}$ and $0.047 / 0.0129=3.6 \mathrm{cpd} / \mathrm{kg} / \mathrm{keV}$, respectively. Thus, that fit produces two "big" contributions, much larger (even orders of magnitude) than their correct estimates 
(see above and Table 1) and much larger than the measurements as well. We remind that the single-hit total rate of the DAMA detectors is around $1 \mathrm{cpd} / \mathrm{kg} / \mathrm{keV}$ (see e.g. [1]).

\subsection{Davis [9] reports (at least) three orders of magnitude wrong estimates}

Taking into account the mean free path (conservatively about $2.6 \mathrm{~m}$ ) of the muon-induced neutrons, the author of [9] estimates: "the effective volume over which these neutrons are produced and still reach the detector to be ...450 $\mathrm{m}^{3}$ ".

This effective volume is wrong by several orders of magnitude, because the author of [9] does not take into account e.g. the geometrical efficiency in DAMA/LIBRA for the detection of those produced neutrons. This effective volume has been properly calculated above: $V_{\text {eff }} \simeq 0.7 \mathrm{~m}^{3}$.

In addition, as reported in Table 1 , the induced modulation amplitudes from neutrons induced by muons and by neutrinos are $\ll 9 \times 10^{-6} \mathrm{cpd} / \mathrm{kg} / \mathrm{keV}\left(\ll 2 \times 10^{-5} \mathrm{cpd} / \mathrm{kg} / \mathrm{keV}\right.$ for neutrons produced in the lead shield) and $\ll 2 \times 10^{-6}$ $\mathrm{cpd} / \mathrm{kg} / \mathrm{keV}$, respectively. These upper limits are orders of magnitude lower than the $A_{\mu}$ and $A_{v}$ values claimed in [9] and mentioned above.

\subsection{DAMA vs solar neutrinos: may DAMA/LIBRA} compete with the multi-hundred-ton experiments for the detection of ${ }^{8} \mathrm{~B}$ solar neutrinos?

The rate for "direct" interactions of solar neutrinos on $\mathrm{NaI}(\mathrm{Tl})$ is around $10^{-5} \mathrm{cpd} / \mathrm{kg} / \mathrm{keV}$ [33] at low energy, many orders of magnitude lower than the measured total single-hit rate in DAMA.

The author of [9] claims that the "indirect" (neutrons induced just by ${ }^{8} \mathrm{~B}$ solar neutrinos) solar neutrino event rate would be $1.17 \mathrm{cpd} / \mathrm{kg} / \mathrm{keV}$ (see above). That is the "indirect" contribution is claimed to be many orders of magnitude larger than the "direct" one; may DAMA/LIBRA compete with multi-hundred-ton experiments for the detection of ${ }^{8} \mathrm{~B}$ neutrinos and even be able to see its annual variation!?

In conclusion, already just considering the arguments given above the claim of [9] is unfounded. Similar outcomes - on the basis of complementary considerations - were also obtained in [34], appeared nearly simultaneously to the preprint of this paper.

\section{Conclusions}

This paper further summarizes in a simple and intuitive way why the neutrons, the muons and the solar neutrinos cannot significantly contribute to the DAMA annual modulation results. In addition, neutrons, muons and solar neutrinos are not a competing background when the DM annual modula- tion signature is investigated since in no case they can mimic this signature. A number of arguments have already been presented in individual papers by DAMA collaboration. Few simple considerations are summarized which demonstrate the incorrectness of the claim reported in [9].

Open Access This article is distributed under the terms of the Creative Commons Attribution License which permits any use, distribution, and reproduction in any medium, provided the original author(s) and the source are credited.

Funded by SCOAP $^{3}$ / License Version CC BY 4.0.

\section{References}

1. R. Bernabei et al., Nucl. Instrum. Methods A 592, 297 (2008)

2. R. Bernabei et al., Eur. Phys. J. C 56, 333 (2008)

3. R. Bernabei et al., Eur. Phys. J. C 67, 39 (2010)

4. R. Bernabei et al., Eur. Phys. J. C 73, 2648 (2013)

5. R. Bernabei et al., La Rivista del Nuovo Cimento 26(1), 1-73 (2003)

6. R. Bernabei et al., Int. J. Mod. Phys. D 13, 2127-2159 (2004)

7. R. Bernabei et al., Eur. Phys. J. C 72, 2064 (2012)

8. R. Bernabei et al., Int. J. Mod. Phys. A 28, 1330022 (2013)

9. J.H. Davis, Phys. Rev. Lett. 113, 081302 (2014). arXiv:1407.1052

10. M. Aglietta et al., hep-ex/9905047

11. M. Aglietta et al., Nuovo Cimento C 12, 467 (1989)

12. D.M. Mei, A. Hime, Phys. Rev. D 73, 053004 (2006)

13. E. Bellotti et al., INFN/TC-85/19 (1985)

14. A. Rindi et al., Nucl. Instrum. Methods A 272, 871 (1988)

15. P. Belli et al., Nuovo Cimento A 101, 959 (1989)

16. R. Aleksan et al., Nucl. Instrum. Methods A 274, 203 (1989)

17. M. Cribier et al., Astropart. Phys. 4, 23 (1995)

18. F. Arneodo et al., Nuovo Cimento A 112, 819 (1999)

19. P. Capuano et al., J. Appl. Geophys. 39, 25 (1998)

20. A. Lindote et al., Astropart. Phys. 31, 366 (2009)

21. M. Ambrosio et al., Astropart. Phys. 7, 109-124 (1997)

22. M. Selvi, On Behalf of the LVD Coll., in Proceedings of the 31st International Cosmic Ray Conference (ICRC'09), Lodz, Poland (2009) (in press)

23. Borexino Coll., in D.D'Angelo Talk at the International Conference Beyond the Desert 2010, Cape Town, South Africa (2010)

24. G. Bellini, Talk at the Scientific Committee II of INFN (2010). https://agenda.infn.it/getFile.py/access? contribId=49\& resId $=0 \&$ materialId $=$ slides $\&$ confId $=2907$

25. Borexino Coll., in Proceedings of International Cosmic Ray Conference (ICRC'11), Beijing, China (2011) (in press). arXiv: 1109.3901

26. Borexino Coll., arXiv:1202.6403 (submitted for publication)

27. M. Ambrosio et al., Astropart. Phys. 19, 313 (2003)

28. K.A. Olive et al., (Particle Data Group), Chin. Phys. C 38, 090001 (2014)

29. M.B. Smy et al., (Super-Kamiokande Collaboration), Phys. Rev. D 69, 011104(R) (2004)

30. J.P. Cravens et al., (Super-Kamiokande Collaboration), Phys. Rev. D 78, 032002 (2008)

31. B. Aharmim et al., (SNO Collaboration), Phys. Rev. D 72, 052010 (2005)

32. G. Bellini et al., (Borexino Collaboration), Phys. Rev. D 89, 112007 (2014)

33. R. Bernabei et al., Astropart. Phys. 4, 45 (1995)

34. P.S. Barbeau et al., arXiv: 1409.3185 